\title{
蛋白激酶 $\mathrm{C}$ 在 $\alpha$ 肾上腺素受体两种亚型 介导血管收缩中的作用比较*
}

\author{
董尔丹 韩启德 张幼怡 禹更生 陈明哲 \\ (北京医科大学第三医院血管医学研究所, 北京 100083)
}

\section{关键词 $\alpha_{1}$ 肾上腺亲受体亚型 蛋白激砤 C 磷酸肌醇代谢 血管收缩}

$\alpha_{1}$ 肾上腺素受体 $\left(\alpha_{1}-A R\right)$ 可以分成 $\alpha_{1 A}$ 和 $\alpha_{1 B}-A R$ 两种亚型, 这两种亚型在大鼠不同血管 的分布有很大差异, 例如主动脉主要含 $\alpha_{1 \mathrm{~B}}-\mathrm{AR}$ 亚型, 肾动脉主要含 $\alpha_{1 \mathrm{~A}}-\mathrm{AR}$ 亚型 ${ }^{[1,2]}$. 我们以 前的工作还证明 $\alpha_{1}-\mathrm{AR}$ 两种亚型激动时都能引起肌醇磷脂水解 ${ }^{[3]}$, 但它们在这两种亚型所介导 血管收缩中所起作用有何差异尚缺乏研究. 本文利用特异性工具药, 在磷酸肌醇 (InsPs) 蓄积 实验和离体灌流血管收缩功能实验中, 以大鼠肾动脉与主动脉中 $\alpha_{1}-\mathrm{AR}$ 分别代表 $\alpha_{1 A}$-与 $\alpha_{1 B}-\mathrm{AR}$ 亚型, 比较蛋白激酶 C(PKC) 在 $\alpha_{1}-\mathrm{AR}$ 两种亚型介导血管收缩中的作用.

\section{1 实验方法}

\section{1 磷酸肌醇蕃积实验}

(1) 去甲肾上腺素 (NE) 介导血管的 InsPs 蓄积作用. 制备大鼠 $5 \mathrm{~mm}$ 长的主动脉和肾动 脉标本, 纵向剖开, 每隔 $1 \mathrm{~mm}$ 剪一切口, 将标本在 $95 \% \mathrm{O}_{2}$ 和 $5 \% \mathrm{CO}_{2}$ 饱和的 $37^{\circ} \mathrm{C} \mathrm{Krebs}$ 液中 温育 $30 \mathrm{~min}$, 冲洗后再将标本放人含 $15 \times 317 \times 10^{10} \mu \mathrm{Bq}{ }^{3} \mathrm{H}$-肌醇的 Krebs 液 $3 \mathrm{~mL}$ 中温育 $1 \mathrm{~h}$, 用 冷 Krebs 液充分冲洗后将标本放人含 $\mathrm{LiCl} 10^{-2} \mathrm{~mol} / \mathrm{L}$ 的 $\mathrm{Krebs}$ 溶液中 $37^{\circ} \mathrm{C}$ 温育 $10 \mathrm{~min}$, 然后 加人不同浓度的 $\mathrm{NE}$ ( 终浓度分别为 $0,0.1,1,10$ 及 $100 \mu \mathrm{mol} / \mathrm{L}$ ), 在含 $\mathrm{LiCl}$ 的 $\mathrm{Krebs}$ 溶液中, 继续 温育 $2 \mathrm{~h}$. 采用柱层析法 ${ }^{[4]}$ 分离并测定 InsPs 蓄积率.

$$
\text { 蓄积率 }(\%)=\frac{\text { 水相总 } \mathrm{CPM}}{\text { 水相总 } \mathrm{CPM}+\text { 脂相总 } \mathrm{CPM}} \times 100 \% \text {. }
$$

（2）氯乙基可乐定对 NE 介导血管 InsPs 蓄积作用的影响. 先将标本置于含氯乙基可乐 定 (Chloroethylclonidine, CEC) $10^{-4} \mathrm{~mol} / \mathrm{L}$ 的经 $95 \% \mathrm{O}_{2}$ 和 $5 \% \mathrm{CO}_{2}$ 气体饱和的 $\mathrm{Krebs}$ 液中 $37^{\circ} \mathrm{C}$ 温育 $30 \mathrm{~min}$, 洗出后再重复上述过程, 所用 $\mathrm{NE}$ 浓度为 $10^{-4} \mathrm{~mol} / \mathrm{L}$.

\section{2 离体血管收缩功能实验}

(1) Staurosporine 对 NE 介导血管收缩的影响. 1 Wistar 大鼠, 体重 $(170 \pm 8) \mathrm{g}$, 以颈椎脱 位法致死后取出所需血管, 制成 $3 \mathrm{~nm}$ 长的环形血管标本, 以 $\mathrm{Han}^{[9]}$ 等报道的方法, 在 $37^{\circ} \mathrm{C}$ 及 通以 $95 \% \mathrm{O}_{2}-5 \% \mathrm{CO}_{2}$ 气体的 $\mathrm{Krebs}$ 溶液中灌流标本, 标本负荷分别为: 主动脉 $1.0 \mathrm{~g}$, 肾动脉 $0.2 \mathrm{~g}$. 在灌流液中加人 $10^{-7} \mathrm{~mol} / \mathrm{L}$ Yohimbine 及 $10^{-6} \mathrm{~mol} / \mathrm{L}$ Propranolol 以分别阻断 $\alpha_{2}$-和 $\beta-$ $\mathrm{AR}$ (在此基础上 $\mathrm{NE}$ 仅激动 $\alpha_{1}-\mathrm{AR}$ ), 加人 $10^{-7} \mathrm{~mol} / \mathrm{L}$ Desmethylimipramine 和 $10^{-6} \mathrm{~mol} / \mathrm{L}$ Normetanephrine 以阻断神经末梢与其它组织对 NE 的摄取. 测定给予不同浓度 NE 时张力的 
改变, 制备累积浓度-收缩效应曲线 $(\mathrm{CRC})$, 并得 $\mathrm{EC}_{50}$, 即收缩达 $50 \%$ 最大效应时 $\mathrm{NE}$ 的浓度 $(\mathrm{mol} / \mathrm{L})$ 并以其负对数值 $p D_{2}$ 表示. 洗出 NE $30 \mathrm{~min}$ 后将标本在含 Staurosporine $10^{-8} \mathrm{~mol} / \mathrm{L}$ 和 $10^{-7} \mathrm{~mol} / \mathrm{L}$ 的 Krebs 液中温育 $30 \mathrm{~min}$, 洗出 Staurosporine 后再重复 NE 的 CRC.

（2）佛波酯对血管的收缩作用. 在上述条件下，观察佛波酯（Phorbol 12-myristate 13acetate, PMA) 对主动脉、肾动脉的直接收缩作用.

\section{3 统计学处理}

所有数据均以均数 \pm 标准误 $(\bar{x} \pm \mathrm{SE})$ 表示, 显著性检验采用 $\mathrm{t}$ 检验或配对 $\mathrm{t}$ 检验.

以上所用试剂除 CEC 为 Research Biochemical Inc 产品, ${ }^{3} \mathrm{H}$-Inositol 为 American Radiolabeled Chemicals 公司产品外, 余均购自 Sigma 公司.

\section{2 结果}

\subsection{NE 介导血管 InsPs 蕃积及 CEC 对其作用的影响}

$\mathrm{NE}$ 能够介导主动脉和肾动脉 InsPs 蓄积增加, 且呈浓度依赖关系．相同浓度的 NE 对主 动脉和肾动脉 InsPs 蓄积的增加作用无显著差异 (表 1). 而经 $\mathrm{CEC} 10^{-4} \mathrm{~mol} / \mathrm{L}$ 温育 $30 \mathrm{~min}$ 后, NE $10^{-4} \mathrm{~mol} / \mathrm{L}$ 对主动脉 InsPs 蓄积的增加作用明显降低, 有非常显著差异, 与基础状态无 差异. 而对肾动脉则影响较小, InsPs 蓄积率仍明显高于基础状态 (表 2).

表 1 去甲肾上腺素诱导大鼠离体主动脉、肾动脉的磷酸肌醇蓄积作用 (以百分率表示) ${ }^{\mathrm{a})}$

\begin{tabular}{|c|c|c|c|c|c|}
\hline \multirow{2}{*}{\multicolumn{2}{|c|}{ 基础状态 (10 例) }} & \multicolumn{4}{|c|}{ 去甲肾上腺素 $/ \mathrm{mol} \cdot \mathrm{L}^{-1}$} \\
\hline & & \multirow{2}{*}{$\frac{10^{-7}(5 \text { 例 })}{38.6 \pm 1.4^{*}}$} & \multirow{2}{*}{$\frac{10^{-6}(5 \text { 例 })}{44.7 \pm 1.0^{* * *}}$} & \multirow{2}{*}{$\frac{10^{-5}(5 \text { 例 })}{49.2 \pm 4.2^{* *}}$} & \multirow{2}{*}{$\frac{10^{-4}(10 \text { 例 })}{51.0 \pm 2.4^{* * *}}$} \\
\hline 主动脉 & $28.0 \pm 3.1$ & & & & \\
\hline 肾动脉 & $19.9 \pm 2.2$ & $26.7 \pm 3.0$ & $33.7 \pm 3.9 * *$ & $51.5 \pm 4.3^{* * *}$ & $56.6 \pm 3.5^{* * *}$ \\
\hline
\end{tabular}

a) 与基础状态相比: *p<0.05, **p<0.01, ***p<0.001

表 2 氯乙基可乐定对去甲肾上腺素诱导的大鼠离体主动脉、肾动脉磷酸肌醇蓄积作用 (百分率表示) 的影响 ${ }^{\mathrm{a})}$

\begin{tabular}{lcllc}
\hline & 例数 & 基础状态 & 去甲肾上腺素 & 去甲肾上腺素 + 氯乙基可乐定 \\
\hline 主动脉 & 10 & $28.0 \pm 3.1$ & $51.0^{ \pm} 2.4^{* * *}$ & $20.3 \pm 2.3^{+++}$ \\
肾动脉 & 10 & $19.9 \pm 2.2$ & $56.6 \pm 3.5^{* * *}$ & $40.2 \pm 4.0^{+* * *}$ \\
\hline
\end{tabular}

a) 与基础状态相比: ${ }^{* * *} p<0.001$, 与去甲肾上腺素相比: $+p<0.05,+++p<0.001$

\subsection{Staurosporine 对 $\alpha_{1}-\mathrm{AR}$ 介导主动脉、肾动脉收缩效应的影响}

Staurosporine $10^{-7} \mathrm{~mol} / \mathrm{L}$ 温育后, $\mathrm{NE}$ 激动 $\alpha_{1}-\mathrm{AR}$ 引起主动脉收缩的CRC 显著右移, $\mathrm{EC}_{50}$

表 3 Staurosporine 对去甲肾上腺素诱导大鼠离体主动脉、肾动脉收缩功能的影响 ${ }^{a)}$

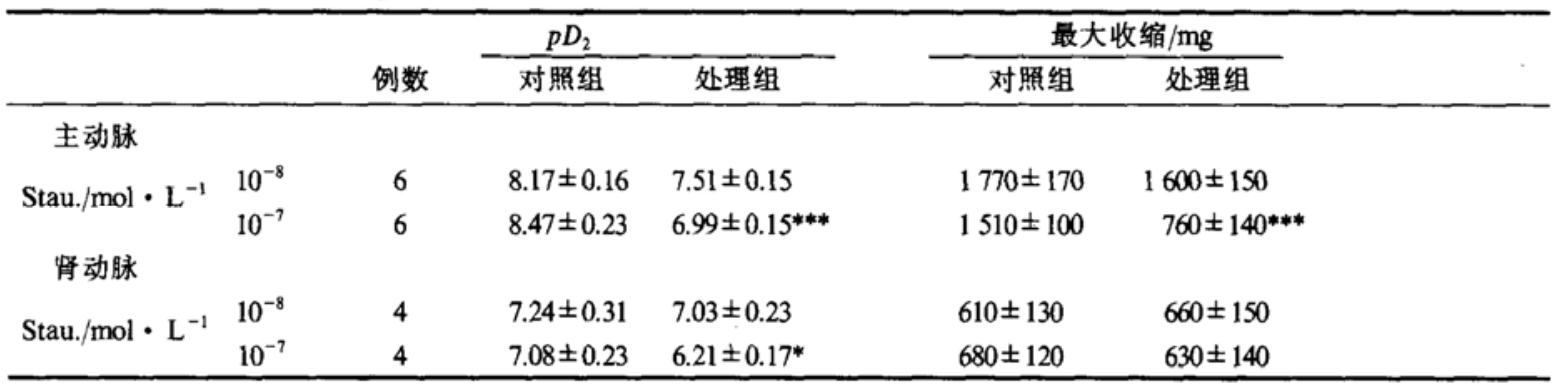

a) 与对照组相比: ${ }^{*} p<0.05,{ }^{* * *} p<0.001$ 
增加了 $36.3 \pm 9.4$ 倍, 最大收缩降低了 $(50.7 \pm 7.2) \%$; 肾动脉经温育后, NE 介导收缩的 CRC 也 显著右移, 但 $\mathrm{EC}_{50}$ 仅增加了 $9.9 \pm 3.7$ 倍, 且最大收缩与对照比无显著差异 (表 3).

\subsection{PMA 对血管的收缩作用}

PMA 剂量依赖性能诱导主动脉产生缓慢、持久的收缩, 而对肾动脉则无作用 (表 4).

\section{3 讨论}

表 4 佛波脂对大鼠离体主动脉、肾动脉的收缩作用比较

我们曾在收缩功能实验中证明大 鼠肾动脉与主动脉中的 $\alpha_{1}-\mathrm{AR}$ 分属 $\alpha_{1 \mathrm{~A}}$-与 $\alpha_{1 \mathrm{~B}}-\mathrm{AR}$ 亚型 ${ }^{[2]}$. 本实验显示 $\mathrm{NE}$ 可浓度依赖性地引起离体大鼠肾动脉 与主动脉 InsPs 的蓄积, 两者间并无显 著差别. 但经 CEC 处理后 NE 介导的

\begin{tabular}{ccc}
\hline & 例数 & 收缩幅度 $/ \mathrm{mg}$ \\
\hline 主动脉 & & \\
$10^{-6} \mathrm{~mol} / \mathrm{L}$ & 6 & $260 \pm 70$ \\
$10^{-5} \mathrm{~mol} / \mathrm{L}$ & 9 & $1200 \pm 160$ \\
肾动脉 & & \\
$10^{-6} \mathrm{~mol} / \mathrm{L}$ & 6 & 0 \\
$10^{-5} \mathrm{~mol} / \mathrm{L}$ & 6 & 0 \\
\hline
\end{tabular}
InsPs 蓄积作用在主动脉明显降低, 同基础状态无明显差异; 而在肾动脉仍明显高于基础状 态, 因而从信息传导方面再次证明肾动脉与主动脉中分别含有 $\alpha_{1 A}-$ 与 $\alpha_{1 B}-A R$ 亚型.

肌醇磷脂水解时除生成InsPs 外还产生二酰基甘油 (DAG), DAG 可进一步激活 PKC, 而 PKC在不同亚型 $\alpha_{1}-\mathrm{AR}$ 引起的血管收缩作用中有何差别则至今未见报道. 本实验显示 PKC 抑制剂 Staurosporine 能显著抑制主动脉 $\alpha_{1}-A R$ 介导的收缩效应, 而对肾动脉 $\alpha_{1}-A R$ 介导的 收缩效应则影响很小. 此外, PKC 激动剂佛波脂能引起主动脉缓慢而强大、持久的收缩, 却不能 引起肾动脉收缩. 上述结果提示在 $\alpha_{1 \mathrm{~B}}-\mathrm{AR}$ 亚型介导的血管收缩效应中 PKC 的激动发挥重 要作用; 但在 $\alpha_{1 \mathrm{~B}}-\mathrm{AR}$ 亚型介导血管收缩的机制中则无 PKC 的参与.

本实验仅采用工具药物观察 PKC 在 $\alpha_{1}-\mathrm{AR}$ 收缩效应中的作用, 具有局限性, 需要进一步 直接测定 $\alpha_{1}-\mathrm{AR}$ 两种亚型激动或给予佛波脂时 DAG 与 PKC 的活性改变, 才能得出定论.

\section{参考文献}

1 Han C, Abel P W, Minneman K P. $\alpha_{1}$-adrenoceptor subtypes linked to different mechanisms for increasing intracellular $\mathrm{Ca}^{2+}$ in smooth muscle. Nature, 1987, 329:333 335

2 Han C, Li J L, Minneman K P. Subtypes of $\alpha_{1}$-adrenoceptors in rat blood vessels. Eur J Pharmacol, 1990, 190: $97 \sim 104$

3 Han C, Wilson K M, Minneman K P. $\alpha_{1}$-Adrenergic receptor subtypes and formation of inositol phosphates in dispersed hepatocytes and renal cells. Mol Pharmacol, 1990, 37: 903 910

4 Han Q D, Wu J H, Yang L H et al. The two subtypes of $\alpha_{1}$-adrenergic receptor existing in rat heart. Chin Sci Bull, 1991, 36: $743 \sim 747$

5 Han C, Abel P W. Neuropeptide Y potentiates contraction and inhibits relaxation of rabbit coronary arteries. J Cardiovas Pharmacol, 1987, 9:675 681 